\title{
A Review of Emotion Recognition Based on EEG using DEAP Dataset Rama Chaudhary ${ }^{1}$, Ram Avtar Jaswal ${ }^{2}$ \\ M. Tech Scholar ${ }^{1}$, Assistant Professor ${ }^{2}$, Department of Electrical Engineering ${ }^{1,2}$ \\ University Institute of Engineering and Technology ${ }^{1,2}$ \\ Kurukshetra, India ${ }^{1,2}$
}

\begin{abstract}
Article Info

Volume 8, Issue 3

Page Number: 298-303

Publication Issue :

May-June-2021

Article History

Accepted : 01 June 2021

Published: 05 June 2021

In modern time, the human-machine interaction technology has been developed so much for recognizing human emotional states depending on physiological signals. The emotional states of human can be recognized by using facial expressions, but sometimes it doesn't give accurate results. For example, if we detect the accuracy of facial expression of sad person, then it will not give fully satisfied result because sad expression also include frustration, irritation, anger, etc. therefore, it will not be possible to determine the particular expression. Therefore, emotion recognition using Electroencephalogram (EEG), Electrocardiogram (ECG) has gained so much attraction because these are based on brain and heart signals respectively. So, after analyzing all the factors, it is decided to recognize emotional states based on EEG using DEAP Dataset. So that, the better accuracy can be achieved.
\end{abstract}

Keywords : Electroencephalogram (EEG), Emotion Recognition, DEAP Dataset, Features Extraction, Artificial Neural Network (ANN)

\section{INTRODUCTION}

Emotions play an important role in the life of human beings. Without emotions, the person can be considered as dead. Emotions are feelings such as hate, rage, affection, fear, confidence, panic, sorrow, joy, depressions, tense, bore, excited, etc. Emotions can be conveyed by facial expressions, by talking, by sign language which help people to interact with one another. There are 2 opinions about emotions: one approach considers emotions as general states of individuals and the other one knows emotions as physiological interactions [1]. Emotions are biochemical states that are linked to all of the body's functions. When it comes to categorising human emotions, there are two common principles to follow: the discrete basic emotion classification and the dimension approaches. According to the discrete basic emotion description approach, emotions can be classified as, positive and negative emotions. 


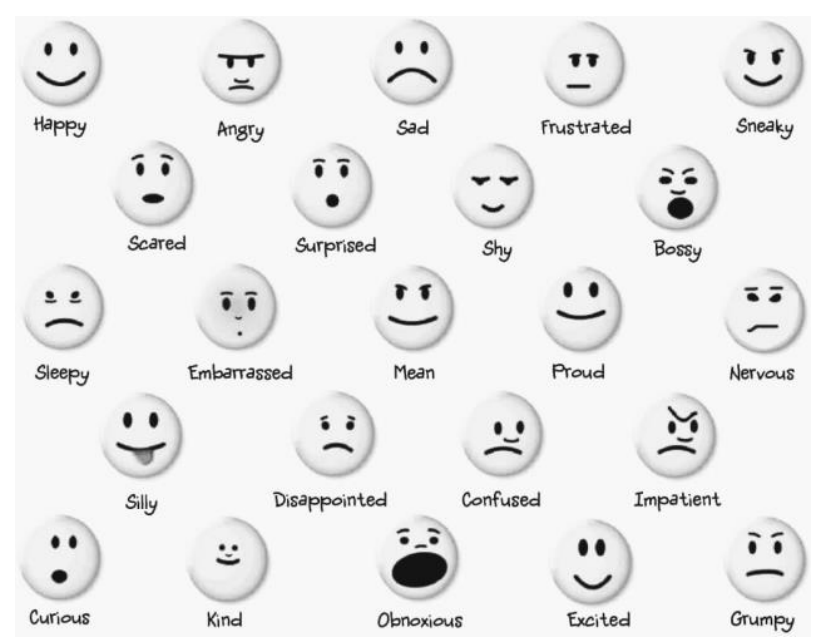

Figure 1: Types of Emotions

Positive emotions are those that make you happy, excited, respecting and loving others, and also making proud of yourself. Some positive emotions are joy, love, excited, happy, pride, garatitude, delighted, etc. Negative emotions are those emotions that make you feel sad, helpless and embarrassed. Some negative emotions are anger, fear, tired, embarrassment, depression, panic, nervous, frustration, irritation, etc. According to dimension approach, the emotions can be classified into three dimensions (valence, arousal \& dominance). Valence describes the extent to which an emotion is positive or negative. Therefore, it is divided into two categories: Positive or high valence emotion (joy, happy, excited, delighted, etc.) and Negative or low valence emotion (fear, anger, sadness, frustrated, etc.).

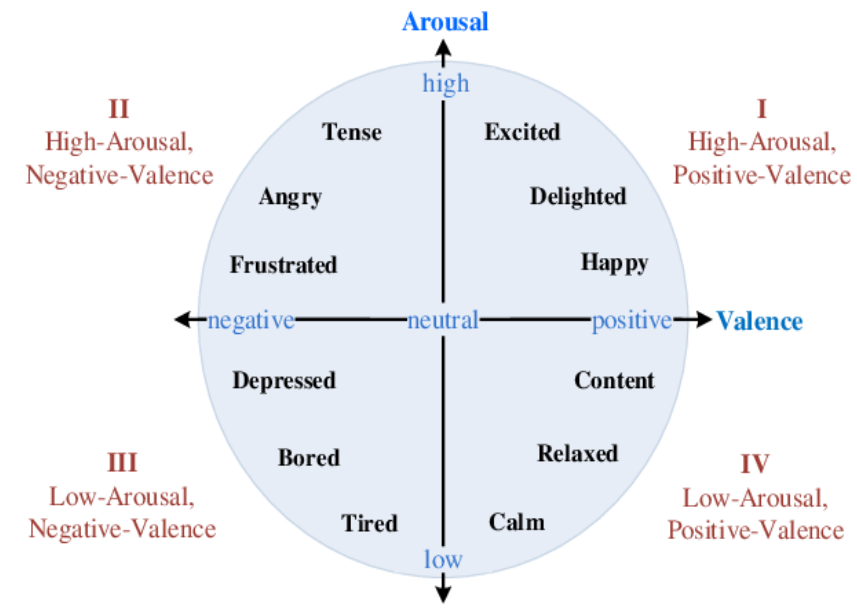

Figure 2: Two dimensional valence-arousal space [20]
Arousal describes the level of excitement or apathy of emotion i.e. it refers to the strength of associated emotional state. It is divided into two categories: Positive or high arousal emotion (angry, tense, happy, excited, etc.) and Negative or low arousal emotion (relaxed, calm, bored, depressed, etc.). The dominance scale ranges from submissive (no control) to dominance (empowered). The DominanceSubmissiveness Scale measures how controlling and dominant you are compared to how controlled and submissive you are. While fear and rage are both unpleasant feelings, anger is the dominating emotion, whilst fear is the submissive emotion.

Brain is the command centre of human nervous system [2]. It controls all the body functions and activities. It consists of mainly three parts: cerebellum, cerebrum and brain stem. Cerebrum is the main and greatest component of human brain and it is divided into left and right hemispheres. Each hemisphere consists of four lobes: frontal, temporal, parietal and occipital lobe. The frontal lobe controls the body movement, personality, concentration, etc. The temporal lobe controls emotions like thirst, hunger, recognizing faces [2] and also controls hearing. The parietal lobe controls the sensation feeling of touch, taste, etc. With the help of occipital lobe, human beings are able to recognize colors, shape, size, depth, distance, etc. The sectional part of human brain is shown in figure 3.

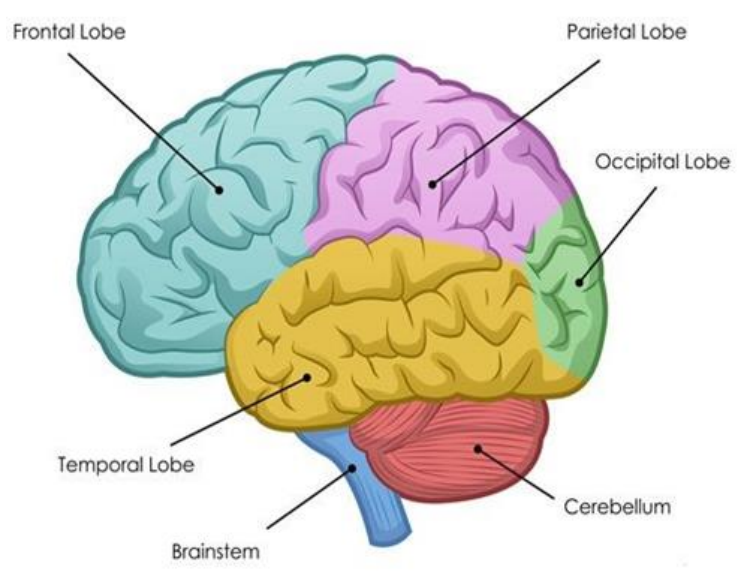

Figure 3 : Human Brain 
This paper is organized as follows: firstly, the information about Electroencephalography has been provided. In next section, the existing work on emotion recognition based on EEG has been discussed which also includes the summary of some existing works based on DEAP dataset. Then, the methodology of proposed work is described which includes information about DEAP Dataset and Artificial Neural Network (ANN). At last, the proposed work is concluded.

\section{ELECTROENCEPHALOGRAPHY}

Electroencephalography is a means of recording electrical activity in the brain using electrophysiological monitoring. Electroencephalogram (EEG) [3] is a term used in medicine to describe the monitoring of the brain's spontaneous electrical activity over time using several electrodes mounted on the scalp. EEG tests the voltage changes which cause due to the flow of ionic current in the neurons of brain. The electrical potential produced by neurons when they are active is recorded using an EEG machine. Small flat metal discs called electrodes are attached to the scalp with wires. These electrodes track electrical impulses in brain and transmit signals to computer for recording the results. In an EEG recording, the electrical impulses appear as wavy lines with peaks and valleys as shown in figure 4. The central nervous system (CNS) generates EEG signals, which react to emotional changes faster than other peripheral neural signals. EEG is one of most important instrument for measuring brain activity. The EEG, on the other hand, does not necessarily represent the electrical activity of a single neuron, but rather the electrical activity of a group of neurons in the brain region where the EEG measurement electrode is located. As a result, the EEG signal contains a wealth of relevant and useful psychophysiological data. EEG signal has a low amplitude of 50 to $200 \mu \mathrm{V}$ and its frequency spectrum is $0.5-100 \mathrm{~Hz}$ although the low frequency range of $0.5-30 \mathrm{~Hz}$ is most important to cognition. Researchers usually break it down into five frequency sub-bands namely $\delta(1-4 \mathrm{~Hz}), \theta(4-8 \mathrm{~Hz}), \alpha(8-13 \mathrm{~Hz})$, $\beta(13-30 \mathrm{~Hz}), \gamma(>30 \mathrm{~Hz})$ [4], each of which corresponds to a different cognitive function.

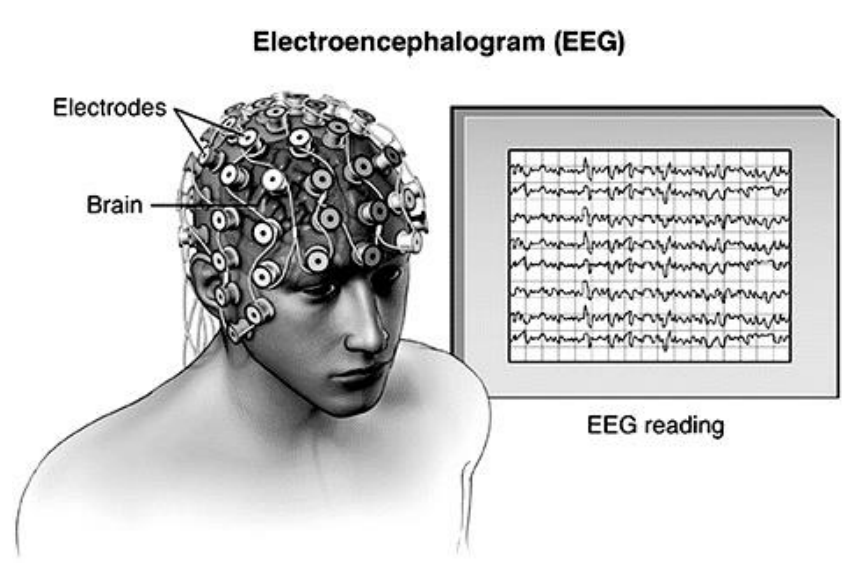

Figure 4: Electroencephalogram

\section{RELATED WORK}

Chao et al. [9], proposed a global feature extraction method that encapsulates the multichannel EEG signals into gray images. First of all, the maximal information coefficient (MIC) was measured for all channels. The spatial characteristics and global interchannel synchronisation features were then extracted from the constructed feature images using a deep learning model with two principal component analysis convolutional layers and a nonlinear transformation operation, which were then fed into support vector machines to perform the emotion recognition tasks. They used DEAP Dataset for collecting the data of human emotional states and accuracy achieved for valence and arousal is $70.21 \%$ and $71.85 \%$ respectively. X. Chen et al. [10], proposed a hierarchical bidirectional Gated Recurrent Unit (GRU) network with attention for classification of human emotions from continues electroencephalogram (EEG) signals. The model's structure is similar to the hierarchical organisation of 
EEG signals. To assess the model's performance, they conducted cross-subject emotion classification trials using the DEAP data set. The experimental results obtained for valence and arousal dimensions are $67.9 \%$ and $66.5 \%$ respectively. Vaishali M. Joshi, Rajesh B. Ghongade [11], used Modified Differential Entropy (MD-DE) feature extractor to detect non-linearity and non-gaussianity of the EEG signal. They used both approaches by analysing 14 patients' EEGs using their own database, named as "IDEA-intellect database for emotion analysis." The bidirectional long short-term memory (BiLSTM) network and the multilayer perceptron (MLP) network are utilised in this study to classify the subjects' emotional states of mind. They used DEAP and SEED dataset also. The accuracy achieved on DEAP dataset for valence and arousal is $73.5 \%$ and $75 \%$ respectively. S. Hwang et al. [12], proposed a novel EEG-based emotion recognition approach that addresses the challenging issue of the subject-dependency. They devised a multi-task deep neural network to handle the challenge. They used a leave-one-subject-out methodology to test the method for classifying EEG emotional labels on the SEED dataset, which contains EEG recordings from 15 subjects and three emotional labels: positive, negative, and neutral. They compared their method to a multi-task deep neural network and a single-task deep neural network that classifies emotional labels with subject labels. The accuracy obtained for both valence and arousal is $64.9 \%$. S. D. You and C. Liu [13], used EEG recordings to assess whether a user likes a video clip he/she is watching. They used DEAP dataset for collecting the human emotional states data. Before categorization, the EEG signals are segmented and characteristics from each segment are computed. K-nearest neighbour, support vector machine, multilayer neural network, and autoencoder neural networks are used as classifiers. The accuracy obtained for valence and arousal on DEAP dataset is $81.10 \%$ and $74.38 \%$ respectively. Parui et al. [14], extracted several features from EEG brain signals and then optimize the set of features using the correlation matrix, information gain calculation, and recursive feature elimination method. The classified data is then based on the optimum feature set. The Extreme Gradient Boosting (XGBoost) algorithm was used for the classification strategy. The XGBoost technique uses the Gradient Boosting framework and is based on the linear model solver and tree learning concepts. The DEAP dataset was used to test this research method. It has also been compared to a variety of classification algorithms, including Naive Bayes, KNN, C4.5, Decision Tree, and Random Forest. The results obtained for valence and arousal are 77.19 and 79.06 respectively. Y. Zhan et al. [15], extracted power spectral features from four frequency bands $(\theta, \alpha, \beta, \gamma)$ and transformed obtained features into cortex-like frames while preserving spatial information of electrodes position, so that the multi-channel, multifrequency bands and time series EEG signals can be efficiently represented. Then, to learn spatial representation from labelled frames, they created a shallow depthwise parallel CNN inspired by the Mobilenet approach. They used DEAP database using segment-level emotion recognition studies. They achieved the competitive accuracy of $84.07 \%$ and $82.95 \%$ on arousal and valence respectively. Pandey P., Seeja K. [16], exploited the image processing capability of convolutional neural network (CNN) and proposed a CNN model to classify different emotions from the scalogram images of EEG data. The continuous wavelet transform was used to create scalogram images from EEG data. Their model is subject-independent, with the goal of extracting emotion-specific features from EEG data regardless of the data source. They used two public databases namely DEAP and SEED. The cross data base criteria were also employed for evaluation in order to show the model as entirely subject independent. The resulted accuracy on DEAP dataset is $61.5 \%$ and $58.5 \%$ for valence and arousal respectively. Eun Jeong Choi, Dong Keun Kim [17], proposed arousal and valence classification obtained from DEAP Dataset using electrocardiography (EEG). The experiments 
used long short-term memory (LSTM) as a deep learning method and were based on 10 prominent central and peripheral nervous system data points. A two-dimensional coordinate plane was used to classify and show arousal and valence. According to the error rate, profiles were created based on the number of hidden layers, nodes, and hyperparameters. The experimental results show an arousal and valence classification model accuracy of 74.65 and 78\%, respectively. Alhagry et al. [18], proposed deep learning method to recognize emotion from raw EEG signals. Long-Short Term Memory (LSTM) is utilised to learn properties from EEG signals, which are subsequently classified into low/high arousal, valence, and liking by the dense layer. The DEAP dataset is utilised to validate this method, which gives an average accuracy of 85.65 percent for arousal, 85.45 percent for valence, and 87.99 percent for liking classes, respectively. $\mathrm{N}$. Zhuang et al. [19], introduced a method for feature extraction and emotion recognition based on empirical mode decomposition (EMD). EEG data are automatically divided into Intrinsic Mode Functions (IMFs) using EMD. The first difference of time series, the first difference of phase, and the normalised energy are all aspects of IMF multidimensional information. This method's effectiveness is tested against a publicly accessible emotional database. The resulted accuracy for valence and arousal is $69.10 \%$ and $71.99 \%$ respectively. Aggarwal et al. [21] recognized human emotional states on EEG signals. They used two GBM's (XGBoost and LightGBM) for emotion classification on DEAP Dataset. The accuracy achieved for valence and arousal is $77.11 \%$ and $60.25 \%$ respectively. After analyzing the previously discussed literature reviews, the proposed work will be based on EEG signal. The DEAP dataset will be used to collect the data of human emotional states and Artificial Neural Network (ANN) will be used as classifier to get the better accuracy.

Table 1: Summary of some existing works on DEAP Dataset

\begin{tabular}{|l|l|l|l|l|}
\hline Reference & Dataset & Classifier & Accuracy (\%) \\
\cline { 4 - 5 } & & & Valence & Arousal \\
\hline Chao et al.[9] & DEAP & SVM & 70.21 & 71.85 \\
\hline X. Chen et al. [10] & DEAP & H-ATT-BGRU & 67.9 & 66.5 \\
\hline Vaishali and Rajesh [11] & DEAP & BiLSTM & 73.5 & 75 \\
\hline You and Liu[13] & DEAP & SAE+LSTM & 81.10 & 74.38 \\
\hline Parui et al.[14] & DEAP & $\begin{array}{l}\text { XGBoost+LightGBM+ } \\
\text { Random forest }\end{array}$ & 77.19 & 79.06 \\
\hline Zhan et al.[15] & DEAP & CNN & 82.95 & 84.07 \\
\hline Pandey and Seeja[16] & DEAP & CNN & 61.50 & 58.50 \\
\hline $\begin{array}{l}\text { Eun Jeong Choi, Dong } \\
\text { Keun Kim [17] }\end{array}$ & DEAP & LSTM & 78 & 74.65 \\
\hline Alhagry et al.[18] & DEAP & LSTM-RNN & 85.45 & 85.65 \\
\hline Zhuang et al. [19] & DEAP & SVM & 69.10 & 71.99 \\
\hline Aggarwal et al. [21] & DEAP & XGBoost+LightGBM & 77.11 & 60.25 \\
\hline
\end{tabular}




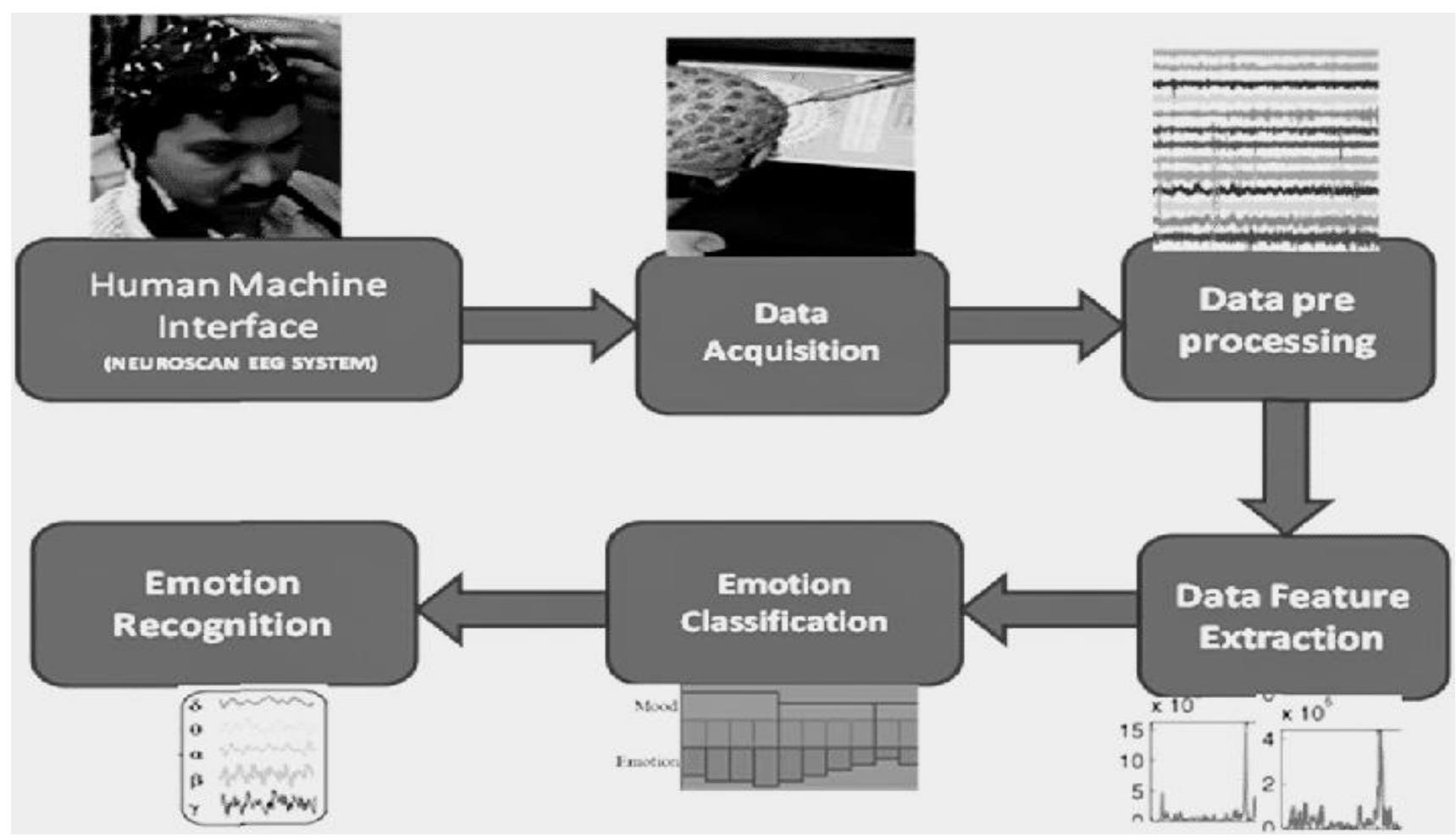

Figure 5 : Block diagram for Emotion Recognition using EEG Signal

\section{METHODOLOGY OF PROPOSED WORK}

To recognize human emotional states on better accuracy, the DEAP Dataset and Artificial Neural Network will be used. So firstly, the data will be collected from DEAP dataset, then it will be preprocessed. After pre-processing, the time domain features will be extracted, then artificial neural network will be used to classify emotions and finally the accuracy will be achieved. The block diagram for this whole process is shown in figure 5 . The methodology for proposed work is discussed below:

\section{A. DEAP Dataset}

The DEAP dataset is a publicly available multimodal dataset [5] that is used to study human affective states. It shows four types of states: valence, arousal, dominance \& liking. Since different sampling rates were used in data collection and different types of tests were performed, the DEAP Dataset is an aggregate of different types of data. The large volume of data is obtained by combining this data into a single comprehensive dataset. The researchers use it for testing the emotion states. For using this dataset,
EULA (End User License Agreement) is to be printed, signed and then upload it via dataset request form. After following all these steps, Username \& Password is to be provided by them. The DEAP dataset [5] was collected from 32 subjects when they were watching 40 sets of 1-min music and video clips. After finishing each video, each participant was asked to give the rating in terms of valance, arousal, dominance and familiarity ranges from 1 to 9 and liking ranges from 1 to 5 . For 22 participants, frontal face video was also recorded. Various signals, such as EEG, electromyograms, breathing region, plethysmographs, temperature, and so on, were reported as 40 channel data during each subject's 40 trials. EEG data is stored in 32 channels out of 40, which are selected for experiments. There are 32 files, one file for each participant's recordings. Each trial contains $63 \mathrm{~s}$ signals, the first $3 \mathrm{~s}$ are the base line signals. The data were down-sampled from $512 \mathrm{~Hz}$ to $128 \mathrm{~Hz}$, and a bandpass frequency filter from $4.0-45.0 \mathrm{~Hz}$ was applied. The frequency bands are decomposed into $\delta(1-4 \mathrm{~Hz}), \theta(4-8 \mathrm{~Hz}), \alpha(8-13 \mathrm{~Hz}), \beta(13-30 \mathrm{~Hz})$, $\gamma(>30 \mathrm{~Hz})[4]$. After collecting and pre-processing the data, five time domain features namely correlation, average, variance, kurtosis and skewness 
will be calculated for separate frequency bands and for different emotional states. In proposed work, the accuracy for two emotional states, valence and arousal, will be calculated.

\section{B. Artificial Neural Network}

An artificial neural network (ANN) is a component of a computational system that simulates how the human brain analyses and processes data. Artificial intelligence (AI) is built on this basis, and it solves problems that would be impossible or difficult to solve by human or statistical standards. An artificial neural network (ANN) is made up of hundreds or thousands of artificial neurons called processing units that are linked by nodes [6]. Input and output units make up these processing units. Input unit contains the given information and then the artificial neural network learn about the information provided in order to generate output. The network is made up of connections, each of which serves as an input to another neuron by passing the output of one neuron.

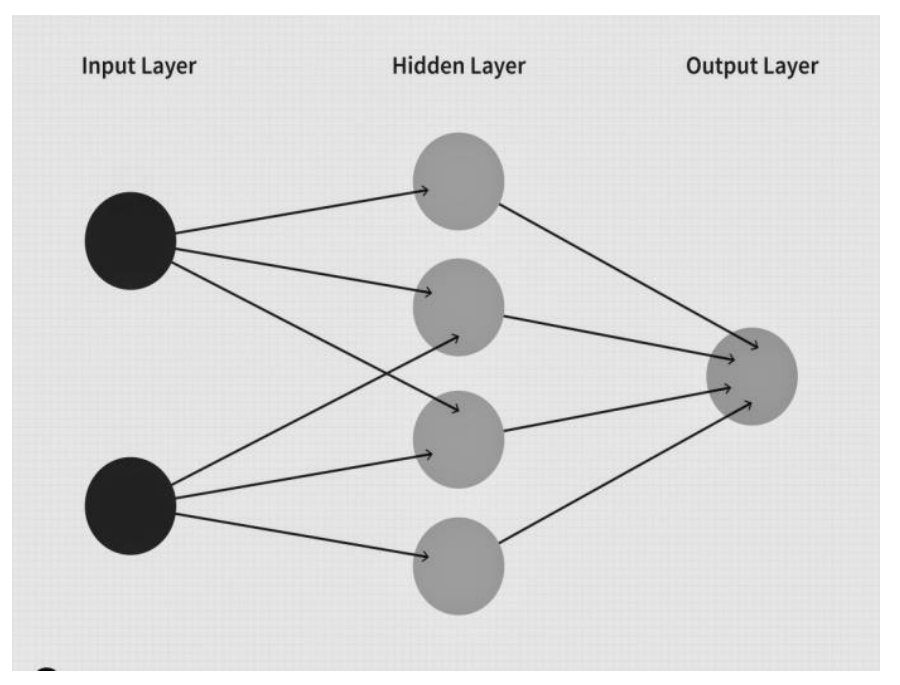

Figure 6: Artificial Neural Network Structure

The input layer, hidden layer, and output layer are the three layers that make up a neural network, as shown in figure 6. The number of hidden layers can be any number, and the number of layers is raised to improve the accuracy and identification rate. The output of feature extraction can be applied to input layers with $\mathrm{N}$ nodes [7]. The number of output nodes can be as many as the number of output classes required. There are three phases in neural network: training phase, validation phase and testing phase. Training phase is used to train the data and it also reduces the data size to few selected important features required from entire data [8]. Validation phase is a separate section of dataset that is used during training to check the performance of model on those data which are not used in training. Testing phase is used to test the final performance of model i.e. how well will it do in production.

\section{CONCLUSION}

Lot of works have been examined based on EEG for recognizing human emotional states. Different analysis methods, classifiers and datasets are used for achieving the better accuracy. After analyzing all works, it is decided to use DEAP Dataset for collecting the data of human emotional states and Artificial Neural Network (ANN) for classifying the emotions so that better accuracy can be achieved without increasing so much complexity. So, the aim of proposed work is to determine best accuracy on less complicated structure.

\section{REFERENCES}

[1]. Thagard P. Mind: Introduction to Cognitive Science. Cambridge, MA: MIT press; 2005.

[2]. Ayan Seal, Puthi Prem Nivesh Reddy, Pingali Chaithanya, Arramada Meghana, Kamireddy Jahnavi, Ondrej Krejcar, and Radovan Hudak, 2020, "An EEG Database and Its Initial Benchmark Emotion Classification Performance," Hindawi Computational and Mathematical Methods in Medicine Volume, Article ID 8303465, 14 pages https://doi.org/10.1155/2020/8303465

[3]. P. Nunez and R. Srinivasan, Electric Fields of the Brain: The Neurophysics of EEG, Oxford University Press, 2006.

[4]. Collet C, Vernet-Maury E, Delhomme G, Dittmar A. Autonomic nervous system response 
patterns specificity to basic emotions. J Auton Nerv Syst 1997;62(1-2):4557.

[5]. Koelstra, S.; Muhl, C.; Soleymani, M.; Lee, J.S.; Yazdani, A.; Ebrahimi, T.; Pun, T.; Nijholt, A.; Patras, I., "DEAP: A Database for Emotion Analysis Using Physiological Signals" IEEE Trans. Affect. Comput. 2012, 3, 18-31.

[6]. Yafei Sun, "Neural Networks for Emotion Classifcation”, August 2013.

[7]. Devi Arumugam and Purushothaman, "Emotion Classification Using Facial Expression," International Journal of Advanced Computer Science and Applications, Vol. 2, No.7, 2011.

[8]. Miss Pooja A Choudhari, Dr. M .R. Dixit, "Human Emotion Recognition using Neural Network Technique" International Journal of Engineering Research \& Technology (IJERT) ISSN: 2278-0181 Vol. 5 Issue 04, April-2016, http://www.ijert.org.

[9]. Chao et al., "Improved Deep Feature Learning by Synchronization Measurements for MultiChannel EEG Emotion Recognition" Hindawi Complexity Volume 2020, Article ID 6816502, 15 pages https://doi.org/10.1155/2020/6816502

[10]. X. Chen et al., "A Hierarchical Bidirectional GRU Model With Attention for EEG-Based Emotion Classification" IEEE Access 7, 118530118540, 2019

[11]. Vaishali M. Joshi, Rajesh B. Ghongade, 2020 "IDEA: Intellect Database for Emotion Recognition" Journal of King Saud UniversityComputer and Information Sciences, https://doi.org/10.1016/j.jksuci.2020.10.007.

[12]. S. Hwang et al., "Subject-independent EEG based emotion recognition using adversarial learning," in Proceedings of the 8th International Winter Conference on BrainComputer Interface (BCI), Gangwon, Korea (South), February 2020.

[13]. S. D. You and C. Liu, "Classification of user preference for music videos based on EEG recordings," in Proceedings of the IEEE 2nd Global Conference on Life Sciences andTechnologies (LifeTech), Kyoto, Japan, March 2020.

[14]. Parui et al., "Emotion recognition from EEG signal using XGBoost algorithm," in Proceedings of the IEEE 16th India Council International Conference (INDICON), Rajkot, India, December 2019.

[15]. Y. Zhan et al., "A computation resource friendly convolutional neural network engine for EEG-based emotion recognition," in Proceedings of the IEEE International Conference on Computational Intelligence and Virtual Environments for Measurement Systems and Applications (CIVEMSA), Tianjin, China, June 2019.

[16]. Pandey P., Seeja K., "Subject independent emotion recognition system for people with facial deformity: an EEG based approach" J. Ambient Intell. Hum. Comput., 1-10, 2020

[17]. Eun Jeong Choi, MS, Dong Keun Kim, PhD, "Arousal and Valence Classification Model Based on Long Short-Term Memory and DEAP Data for Mental Healthcare Management" Healthcare Informatics Research 2018;24(4):309-316 DOI: https://doi.org/10.4258/hir.2018.24.4.309

[18]. Alhagry et al., "Emotion Recognition based on EEG using LSTM Recurrent Neural Network", (IJACSA) International Journal of Advanced Computer Science and Applications, Vol. 8, No. 10, 2017

[19]. N. Zhuang et al., "Emotion Recognition from EEG Signals Using Multidimensional Information in EMD Domain", Hindawi BioMed Research International Volume 2017, Article ID 8317357, 9 pages https://doi.org/10.1155/2017/8317357.

[20]. Liang-Chih Yu, Lung-Hao Lee, Shuai Hao, Jin Wang, Yunchao He, Jun Hu, K. Robert Lai and Xuejie Zhang, 2016, "Building Chinese 
Affective Resources in Valence-Arousal

Dimensions," DOI: 10.18653/v1/N16-1066

https://www.researchgate.net/publication/3041

24018

[21]. S. Aggarwal, L. Aggarwal, M. S. Rihal, and S. Aggarwal, "EEG based participant independent emotion classification using gradient boosting machines," in Proceedings of the IEEE 8th International Advance Computing Conference (IACC), pp. 266-271, Greater Noida, India, December 2018.

\section{Cite this article as :}

Rama Chaudhary, Ram Avtar Jaswal" A Review of Emotion Recognition Based on EEG using DEAP Dataset", International Journal of Scientific Research in Science, Engineering and Technology (IJSRSET), Online ISSN : 2394-4099, Print ISSN : 2395-1990, Volume 8 Issue 3, pp. 298-303, May-June 2021. Available

doi : https://doi.org/10.32628/IJSRSET218352

Journal URL : https://ijsrset.com/IJSRSET218352 